\title{
Rational optimization of reprogramming culture conditions for the generation of induced pluripotent stem cells with ultra-high efficiency and fast kinetics
}

\author{
Jiekai Chen ${ }^{1, *}$, Jing Liu ${ }^{1, *}$, You Chen ${ }^{1}$, Jiaqi Yang ${ }^{1}$, Jing Chen ${ }^{1}, \mathrm{He} \mathrm{Liu}^{1}$, Xiangjie Zhao ${ }^{1}$, Kunlun $\mathrm{Mo}^{1}$, \\ Hong Song ${ }^{1}$, Lin Guo ${ }^{1}$, Shilong $\mathrm{Chu}^{1}$, Deping Wang ${ }^{1}$, Ke Ding ${ }^{1}$, Duanqing Pei ${ }^{1}$
}

${ }^{1}$ Key Laboratory of Regenerative Biology, South China Institute for Stem Cell Biology and Regenerative Medicine at Guangzhou Institutes of Biomedicine and Health, Chinese Academy of Sciences, Guangzhou 510530, China

The ectopic expression of several transcription factors can restore embryonic cell fate to cultured somatic cells and generate induced pluripotent stem cells (iPSCs), revealing a previously unknown pathway to pluripotency. However, this technology is currently limited by low efficiency, slow kinetics and multi-factorial requirement. Here we show that reprogramming can be improved and dramatically accelerated by optimizing culture conditions. First, we developed an optimized defined medium, iCD1, which allows Oct4/Sox2/Klf4 (OSK)-mediated reprogramming to achieve ultra-high efficiency $(\mathbf{1 0 \%}$ at day 8$)$. We also found that this optimized condition renders both Sox2 and Klf4 dispensable, although the elimination of these two factors leads to lower efficiency and slower kinetics. Our studies define a shortened route, both in timing and factor requirement, toward pluripotency. This new paradigm not only provides a rationale to further improve iPSC generation but also simplifies the conceptual understanding of reprogramming by defined factors.

Keywords: iPSCs; stem cells; reprogramming; medium; culture condition

Cell Research (2011) 21:884-894. doi:10.1038/cr.2011.51; published online 29 March 2011

\section{Introduction}

Embryonic stem cells (ESCs) and induced pluripotent stem cells (iPSCs) undergo infinite self-renewal in vitro and differentiate into cell types of all three germ-layers both in vitro and in vivo [1-6]. This pluripotent property appears to be regulated by a network of transcription factors, with Oct4, Sox 2 and Nanog at the core [7]. Oct4, which is expressed exclusively in the early embryo and germ cell lineage, was shown to be the master regulator of pluripotency both in vitro and in vivo [8-10]. Sox2 and Nanog are physically associated with Oct4 and target similar downstream genes [7, 11-14]. However, even though Sox2- and Nanognull mice are early embryonic lethal, they die at a later stage

\footnotetext{
*These two authors contributed equally to this work.

Correspondence: Duanqing Pei

Tel: +86-20-3201-5201

E-mail: pei_duanqing@gibh.ac.cn

Received 10 February 2011; revised 23 February 2011; accepted 25 February 2011; published online 29 March 2011
}

than Oct4-null mice $[9,15,16]$. Therefore, it appears that both Sox 2 and Nanog play auxiliary roles in the housekeeping machinery of pluripotency.

In addition to transcription factors, extracellular signaling also plays a critical role in regulating ESC self-renewal and differentiation. The self-renewal of ESCs is known to be highly dependent on culture conditions. Mouse ESCs were first derived and maintained with empirical applications of feeder layers and serum $[1,2]$. Rational improvement of culture conditions greatly promotes mechanistic insight into pluripotency and self-renewal. The serum-free, feeder-free culture of ESCs based on leukemia inhibitory factor (LIF) and bone morphogenetic proteins (BMPs) allows researchers to define extracellular signaling regulation during self-renewal $[13,17,18]$. Accumulated mechanistic insights led to the concept of a ground state for pluripotency and to a rational approach for the derivation and maintenance of mouse and rat ESCs with only two small-molecule inhibitors (2i), MEK1/2 inhibitor (PD0325901) and GSK3 inhibitor (CHIR99021) [19], which is in contrast to the empirical success of the mouse ESC derivation with serum 
and feeders $[1,2]$. This chemically defined condition also allowed for a more precise description of pluripotency transcriptional regulation [20]. Rational optimization for human ESCs also resulted in a chemically defined medium [21, 22], which greatly facilitates mechanistic research of human ESC maintenance and differentiation [23].

Pluripotency lost during differentiation can be recaptured by somatic cell nuclear transfer (SCNT), fusion between somatic cells and ESCs or induction with defined factors (iPS), which reset the epigenetic state of a somatic cell into a pluripotent cell [24]. The iPS approach offers a great opportunity to understand the epigenetic regulation of cell fate determination at the biochemical, molecular and cellular levels triggered by reprogramming factors $[4,25$, 26]. However, it was unexpected that the Yamanaka factors did not include Nanog, but did include Klf4 and Myc, suggesting a mechanistic difference between induction and maintenance of pluripotency [4]. Subsequent studies revealed that Myc is dispensable [27], suggesting that the factor requirement of reprogramming should be further clarified. Most recently, several studies pointed out that a single Oct4 is sufficient to reprogram somatic cells [28-31], opening a gate to explore the reprogramming mechanism based on a one-factor model. We have described that fibroblasts undergo a mesenchymal-to-epithelial transition (MET) during reprogramming, and that TGF $\beta$ signaling, which is present in the serum, inhibits reprogramming by inducing EMT [32]. Therefore, we hypothesized that the reestablishment of pluripotency would be regulated by the extracellular environment and by ectopic expression of transcription factors. Here we show that rational medium optimization would greatly enhance factor-based reprogramming with less factors and faster kinetics.

\section{Results}

\section{Optimization of culture media for reprogramming}

We previously reported that the reprogramming of somatic cells by defined factors is regulated by the extracellular milieu, despite being a nuclear process $[32,33]$. We showed that signals transmitted by TGF $\beta$ in the serum or produced by mouse embroynic fibroblasts (MEFs) are inhibitory to the reprogramming process and function largely by inducing EMT and preventing MET, a recently identified key step for reprogramming $[32,34,35]$. In addition, several small molecules, including vaproic acid (VPA) and ascorbic acid (vitamin $\mathrm{C}$ ), were reported to improve reprogramming or functionally substitute for reprogramming factors [36-40]. These findings suggest that one can formulate an optimized culture system to yield high and optimized reprogramming efficiency.

We chose OKS-mediated reprogramming for medium optimization because the oncogene $c-M y c$ is dispensable and is undesirable due to safety concerns [27, 33, 41]. A commonly used retroviral delivery system is employed here for adaptability and convenient monitoring of transgene silencing, an important criterion for full reprogramming [27, 42]. We designed a workflow to perform stepwise screening of compounds, growth factors and cytokines to optimize culture conditions (Figure 1A).

As illustrated in Figure 1A, we started the optimization process by building a "positive" library of compounds and growth factors through a screening process for their ability to support or inhibit OKS reprogramming in standard ES culture medium or iSF1 [33]. Compounds or growth factors that showed inhibitory effects in both ES and iSF1 systems, such as TGF $\beta$, SB203580 and retinol acetate, were eliminated (Figure 1B). The remaining compounds or growth factors, together with compounds or growth factors reported to enhance reprogramming or sustain pluripotency of ESCs (such as VPA, BIX01294, A83-01 and PD0325901, among others) $[19,37]$, were selected into this "positive" library and used in reiterative runs of subsequent optimization (Figure $1 \mathrm{~A})$

In the meantime, we began formulating a "basal" medium based on current knowledge of serum-free cell culture. A chemically defined DMEM medium supplemented with insulin, transferrin, selenium, nonessential amino acid (NEAA), recombinant bovine serum albumin and progesterone, based on their support for the growth of NIH $3 \mathrm{~T} 3$ cell line [41], was selected as the starting medium as it cannot support the growth of either MEFs or ESCs without supplementing with various growth factors. Therefore, this starting medium would allow the identification of growth factors that can support either the self-renewal of ESCs or the proliferation of MEFs as illustrated in Figure 1A. As such, the addition of the growth factors into this starting medium would allow for the formulation of a medium that can sustain ESC self-renewal and MEF proliferation, two important criteria that we considered critical for OKS-mediated reprogramming as reported previously [33]. As a result, bFGF, lipid mixture, LIF and some commonly used components in serum-free medium (such as vitamins, hormones, lipoic acid, ethanolamine and glutathione) [22, 43] were supplemented into the initial chemically defined medium to satisfy the minimal requirements of cell culture for both MEFs and ESCs (Figure 1A and 1B). This "basal" medium supports reprogramming, albeit with low efficiency and slow kinetics; therefore, this formulation was used in our screening system for small-molecule compounds for further optimization.

Then we began to screen the "positive" library with OKS-mediated reprogramming in "basal" medium and found that vitamin $\mathrm{C}$ can boost reprogramming as expected 
A

Candidate compounds

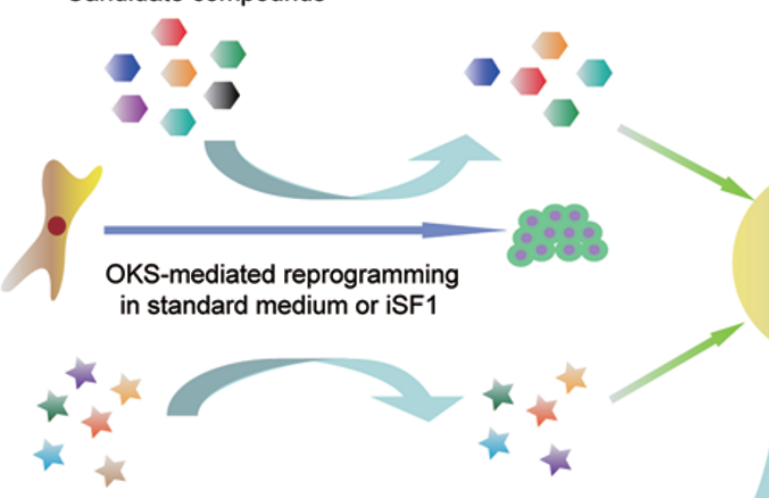

Candidate growth factors

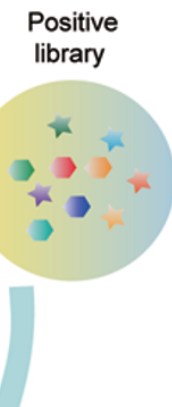

Chemically defined medium (e.g., DMEM) based optimization



MEF proliferation assay
iCD1 medium
Additional rounds of optimization for reprogramming
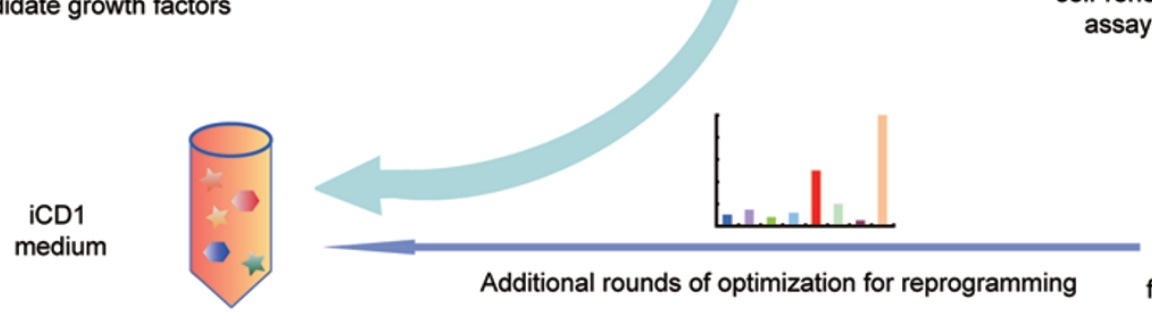

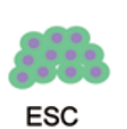
self-renewal assay

"Basal" medium for reprogramming

B

\begin{tabular}{|c|c|c|c|c|c|c|c|c|}
\hline & \multirow{2}{*}{ Components } & \multirow{2}{*}{ Origin } & \multirow{2}{*}{$\begin{array}{c}\text { Functional } \\
\text { Confirmation }\end{array}$} & \multicolumn{3}{|c|}{ OKS-mediated Reprogramming efficiency } & \multirow{2}{*}{$\begin{array}{c}\text { MEFs } \\
\text { proliferation }\end{array}$} & \multirow{2}{*}{$\begin{array}{c}\text { ESCs } \\
\text { pluripotency }\end{array}$} \\
\hline & & & & Based on mES & Based on iSF1 & Based on iCD1 & & \\
\hline \multirow{5}{*}{ Vitamins } & Vitamin C & Sigma & YES & ++ & $+/-$ & +++ & ND & + \\
\hline & D,L-alpha-tocopherol & Sigma & ND & $+/-$ & $+/-$ & $+/-$ & $+/-$ & ND \\
\hline & Thiamine $\mathrm{HCl}$ & Sigma & ND & ND & + & $+/-$ & + & ND \\
\hline & Retinol, all trans & Sigma & ND & $+/-$ & $+/-$ & $+/-$ & ND & ND \\
\hline & Vitamin $\mathrm{B}_{12}$ & Sigma & ND & ND & + & + & + & ND \\
\hline \multirow{11}{*}{$\begin{array}{l}\text { Growth } \\
\text { factors }\end{array}$} & Insulin & Sigma & ND & ND & ++ & +++ & +++ & ND \\
\hline & Transferrin & Sigma & ND & ND & $+/-$ & + & ++ & ND \\
\hline & Catalase & Sigma & ND & ND & $+/-$ & $+/-$ & + & $+/-$ \\
\hline & Superoxide Dismutase & Sigma & ND & ND & + & + & + & $+/-$ \\
\hline & Corticosterone & Sigma & ND & ND & $+/-$ & $+/-$ & + & ND \\
\hline & Progesterone & Sigma & ND & ND & ND & $+/-$ & + & ND \\
\hline & bFGF & Invitrogen & ND & $+/-$ & ++ & +++ & +++ & $+/-$ \\
\hline & TGF beta & R\&D & ND & -- & -- & -- & - & $+/-$ \\
\hline & BMP4 & R\&D & ND & $+/-$ & $+/-$ & - & + & + \\
\hline & LIF & Millipore & ND & + & ++ & ++ & $+/-$ & +++ \\
\hline & Noggin & $R \& D$ & ND & $+/-$ & $+/-$ & $+/-$ & $+/-$ & ND \\
\hline \multirow{10}{*}{$\begin{array}{c}\text { Small } \\
\text { molecules }\end{array}$} & AZA & Sigma & YES & + & $+/-$ & $+/-$ & -- & ND \\
\hline & VPA & EMD & YES & ++ & ++ & $+/-$ & - & - \\
\hline & TSA & Sigma & YES & + & + & ND & -- & - \\
\hline & A83-01 & Sigma & YES & + & + & $+/-$ & $+/-$ & $+/-$ \\
\hline & PD0325901 & in home & YES & -- & --- & -- & --- & ++ \\
\hline & Chir99021 & in home & YES & ++ & ++ & +++ & + & + \\
\hline & LiCl & Sigma & ND & $+/-$ & + & + & + & + \\
\hline & PFT- $a$ & Sigma & ND & $+/-$ & $+/-$ & $+/-$ & + & $+/-$ \\
\hline & Y27632 & EMD & YES & $+/-$ & $+/-$ & $+/-$ & ND & $+/-$ \\
\hline & SB203580 & Sigma & ND & - & - & ND & ND & ND \\
\hline
\end{tabular}

+ : positive effect +/-: not significant -: negative effect ND: not determined
Functional confirmation: these compounds were tested according to their effects reported in the literature MEF proliferation: based on iCD1 ESC pluripotency: based on N2B27

Figure 1 Medium optimization for OKS-mediated reprogramming. (A) The screening strategy for formulating chemically defined iCD1 medium. (B) Information about representative compounds tested in medium optimization. 
[39]. By supplementing the basal medium with vitamin C, we acquired a new starting medium for secondary screening for compounds that may further enhance reprogramming. These secondary screenings by using "basal" medium supplemented with the compound or growth factor, which showed positive effect in previous screenings, were repeated several rounds (Figure 1B) to select a series of compounds that can further boost reprogramming in a sequential fashion. As a result, we established a formulation in which OKS-mediated reprogramming was more efficient than all other combinations tested. In sum, we identified CHIR99021, LiCl, 2-phospho-L-ascorbic acid (this isoform of vitamin $\mathrm{C}$ is critical for its stability), vitamin B12 and thiamine as critical small chemical components of the optimized medium.

Finally, we determined the optimal concentration of all components except for those in basal DMEM, commercial NEAA and lipid mixture, according to their effect on reprogramming (Figure 2). If there was no evidence showing that elimination of the supporting components contained in the "basal" medium would improve the efficiency (Figure 2A-2I), we chose to keep them in the final formulation at the appropriate concentration. As a result, we developed the chemically defined medium iCD1 (for iPS chemically defined medium 1, components and concentrations shown in Table 1), which can support reprogramming much better than other protocols in the literature (Figure 3A and Supplementary information, Figure S1) [4, 33, 39, 44]. We showed that iCD1 delivers a reprogramming efficiency of $\sim 1400$ Oct4-GFP-positive colonies from 15000 starting MEFs on day 8 post-treatment (Figure 3A and 3B), which represents a corrected efficiency of 45 times better than the vitamin C-based protocol [39]. The rapid and robust reprogramming observed in iCD1 was confirmed at the molecular level by analyzing the expression of pluripotent markers, including Nanog, endogenous Oct4 and Dppa3 (Figure 3C and Supplementary information, Figure S2A). By contrast, iCD1 does not induce or improve the expression of reprogram-
A

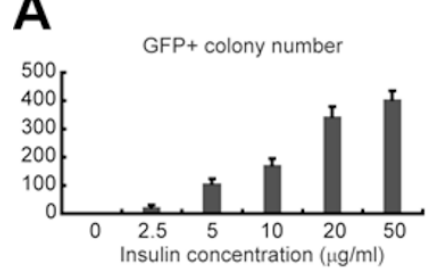

E

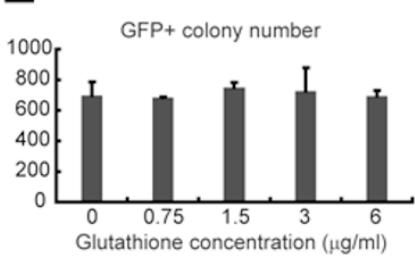

I

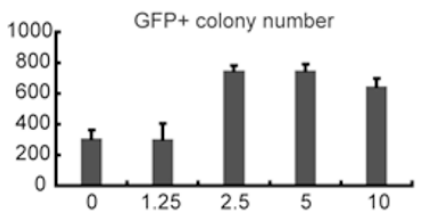

Superoxide dismutase concentration $(\mu \mathrm{g} / \mathrm{ml})$

\section{M}

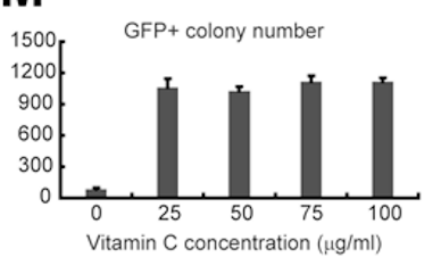

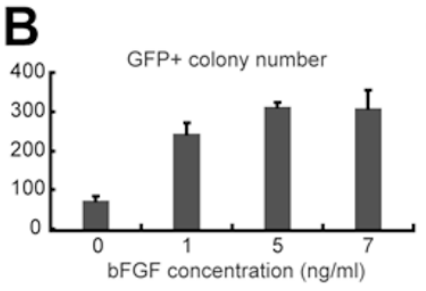

$\mathbf{F}$
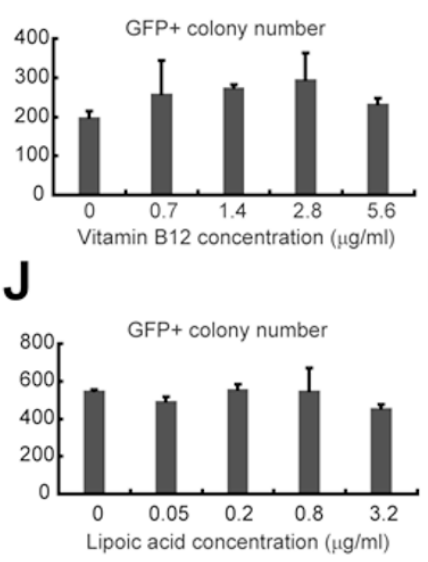

N

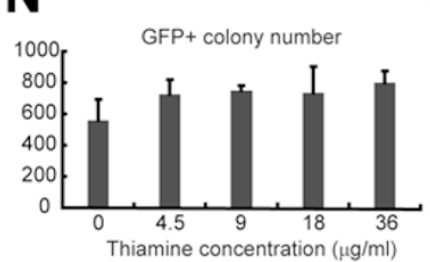

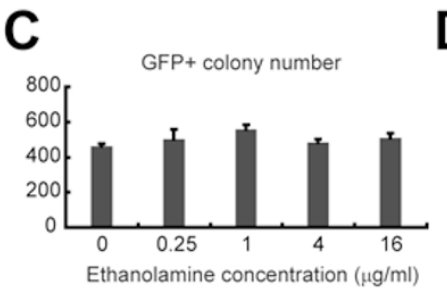

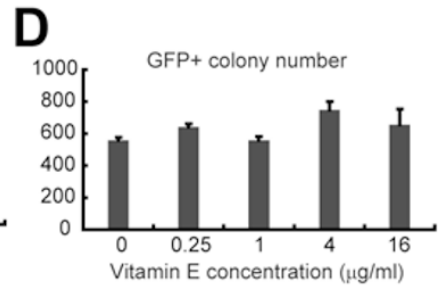

G

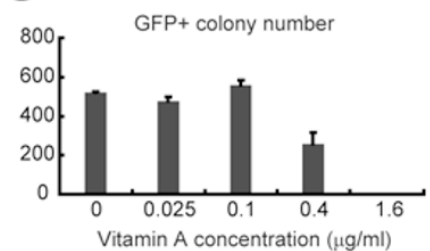

$\mathrm{H}$

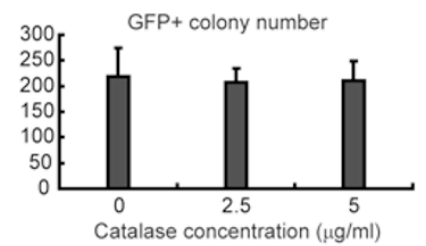

$\mathbf{L}$
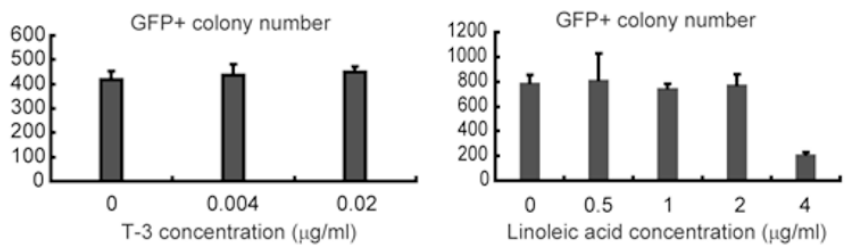

0

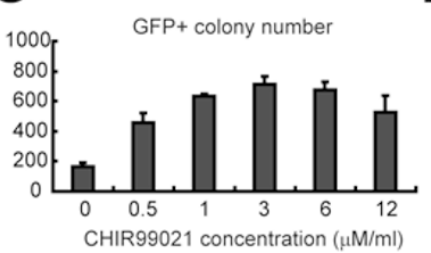

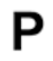

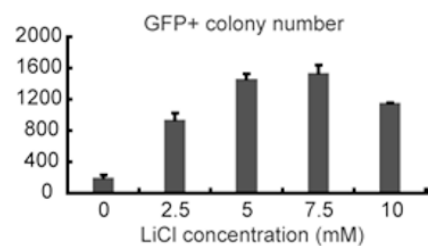

Figure 2 Concentration optimization of components in iCD1. (A-P) The indicated components were supplemented into iCD1 (minus the tested component) at different concentrations, and their effect on OKS-mediated reprogramming was examined ( $n$ = 3). All error bars indicate SD (this is true for all figures unless noted otherwise). 
ming factors in MEF cultures (Supplementary information, Figure S2B), suggesting that iCD1 enhances reprogramming through another mechanism.

Further characterization of $i C D 1$ and application on other cell types

Six components of iCD1 underwent rounds of optimization and were further tested for their contributions to MEF proliferation and reprogramming (Figures 1, 2, 3D and $3 \mathrm{E})$. Except for the commonly used components in chemically defined medium, the effects of bFGF, vitamin $\mathrm{C}$ and GSK3 inhibitors (including $\mathrm{LiCl}$ and CHIR99021) are notable (Figures 2 and 3D and Supplementary information,
Figure S2C). Among them, bFGF is a critical component that supports the proliferation of OKS-transduced MEFs and plays an important role in the early stage of reprogramming (Figure 3E and Supplementary information, Figure $\mathrm{S} 3 \mathrm{~A}$ ). By contrast, vitamin $\mathrm{C}$ enhances iPSC generation in a cell-division-rate-independent manner [45] (Figure 3E). Unexpectedly, known compounds contained in our library, including Kenpaullone, BIX01294, Bayk8644, BMP4, the TGF $\beta$ receptor inhibitor A83-01 and the histone deacetylase (HDAC) inhibitor VPA, which were previously reported to benefit reprogramming, had no effect on iCD1-based OKSmediated reprogramming (Supplementary information, Figure S3B-S3D). We presume that most of their effects are

Table 1 Complete formulation for iCD1 medium

\begin{tabular}{|c|c|c|c|}
\hline INORGANIC SALTS & $\mathrm{mg} / \mathrm{L}$ & AMINO ACIDS & $\mathrm{mg} / \mathrm{L}$ \\
\hline $\mathrm{LiCl}$ & $2.12 \mathrm{E}+02$ & L-Arginine $\cdot \mathrm{HCl}$ & $8.40 \mathrm{E}+01$ \\
\hline $\mathrm{CaCl}_{2}$ (anhyd.) & $2.00 E+02$ & L-Alanine & $8.90 \mathrm{E}+00$ \\
\hline $\mathrm{Fe}\left(\mathrm{NO}_{3}\right)_{3} \cdot 9 \mathrm{H}_{2} \mathrm{O}$ & $1.00 \mathrm{E}-01$ & L-Asparagine & $1.32 \mathrm{E}+01$ \\
\hline $\mathrm{KCl}$ & $4.00 \mathrm{E}+02$ & L-Aspartic acid & $1.33 \mathrm{E}+01$ \\
\hline $\mathrm{MgSO}_{4}$ & $9.77 \mathrm{E}+01$ & L-Cystine $\cdot 2 \mathrm{HCl}$ & $6.30 \mathrm{E}+01$ \\
\hline $\mathrm{NaCl}$ & $6.40 \mathrm{E}+03$ & Glycine & $4.50 \mathrm{E}+01$ \\
\hline $\mathrm{NaHCO}_{3}$ & $3.70 E+03$ & L-Glutamic acid & $1.47 \mathrm{E}+01$ \\
\hline $\mathrm{NaH}_{2} \mathrm{PO}_{4} \cdot \mathrm{H}_{2} \mathrm{O}$ & $1.25 \mathrm{E}+02$ & L-Histidine $\mathrm{HCl} \cdot \mathrm{H}_{2} \mathrm{O}$ & $4.20 \mathrm{E}+01$ \\
\hline Sodium selenite & 1.86E-02 & L-Isoleucine & $1.05 \mathrm{E}+02$ \\
\hline ENERGY SUBSTRATES & & L-Leucine & 1.05E+02 \\
\hline $\begin{array}{l}\text { EINERGY SUDSTRATES } \\
\text { D-Glucose }\end{array}$ & & L-Lystine $\mathrm{HCl}$ & $1.46 \mathrm{E}+02$ \\
\hline D-Glucose & $4.50 \mathrm{E}+03$ & L-Methionine & $3.00 \mathrm{E}+01$ \\
\hline Sodium pyruvate & $1.10 \mathrm{E}+02$ & L-Phenylalanine & $6.60 \mathrm{E}+01$ \\
\hline $\mathrm{D}(+)$-Galactose & $1.50 \mathrm{E}+01$ & L-Proline & $1.15 \mathrm{E}+01$ \\
\hline & & L-Serine & $6.30 \mathrm{E}+01$ \\
\hline LIPIDS & & L-Threonine & $9.50 \mathrm{E}+01$ \\
\hline Arachidonic acid & 2.00E-02 & L-Tryptophan & $1.60 \mathrm{E}+01$ \\
\hline Cholesterol & $2.20 \mathrm{E}+00$ & L-Tyrosine $2 \mathrm{Na} \cdot 2 \mathrm{H}_{2} \mathrm{O}$ & $1.04 \mathrm{E}+02$ \\
\hline linoleic acid & 1.00E-01 & L-Valine & $9.40 E+01$ \\
\hline linolenic acid & 1.00E-01 & & \\
\hline $\begin{array}{l}\text { Myristic acid } \\
\text { Oleic acid }\end{array}$ & $\begin{array}{l}1.00 \mathrm{E}-01 \\
1.00 \mathrm{E}-01\end{array}$ & \multicolumn{2}{|c|}{ GROWTH FACTORS/PROTEINS } \\
\hline Palmitoleic acid & 1.00E-01 & Insulin (Bovine, Recombinant) & $5.00 \mathrm{E}+01$ \\
\hline Palmitic acid & $1.00 \mathrm{E}-01$ & Transferrin(Human) & $1.00 \mathrm{E}+02$ \\
\hline Pluronic F-18 & $1.00 \mathrm{E}+03$ & Recombinant BSA Fraction $\mathrm{V}$ & $1.00 \mathrm{E}+03$ \\
\hline Stearic acid & 1.00E-01 & Catalase & $2.50 \mathrm{E}+0 \mathrm{C}$ \\
\hline Tween 80 & $2.20 \mathrm{E}+01$ & Glutathione (Reduced) & $1.50 \mathrm{E}+0 \mathrm{C}$ \\
\hline VITAMINS & & Superoxide dismutase & $2.50 \mathrm{E}+0 \mathrm{C}$ \\
\hline VIIAMINS & & T-3/Albumin Complex & $2.00 \mathrm{E}-03$ \\
\hline 2-Phospho-L-ascorbic acid & $5.00 E+01$ & Corticosterone & $2.00 \mathrm{E}-02$ \\
\hline D,L-alpha-tocopherol(Vitamin E) & $1.00 \mathrm{E}+00$ & Progesterone & $1.26 \mathrm{E}-02$ \\
\hline D,L-alpha-tocopherol acetatec & $1.00 \mathrm{E}+00$ & basic FGF & $5.00 \mathrm{E}-03$ \\
\hline Biotin & 1.00E-01 & Leukemia inhibitory factor & $1.00 \mathrm{E}-02$ \\
\hline D-Ca pantothenate & $4.00 \mathrm{E}+00$ & & \\
\hline Choline chloride & $4.00 \mathrm{E}+00$ & \multirow{2}{*}{\multicolumn{2}{|c|}{ OTHER COMPONENTS }} \\
\hline Folic acid & $4.00 \mathrm{E}+00$ & & \\
\hline i-Inositol & $7.20 \mathrm{E}+00$ & Putrescine $\cdot 2 \mathrm{HCl}$ & $3.22 \mathrm{E}+01$ \\
\hline Niacinamide & $4.00 \mathrm{E}+00$ & L-Carnitine $\mathrm{HCl}$ & $2.00 E+00$ \\
\hline Pyridoxine $\mathrm{HCl}$ & $4.00 E+00$ & Ethanolamine $\mathrm{HCl}$ & $1.00 \mathrm{E}+00$ \\
\hline Riboflavin & $4.00 \mathrm{E}-01$ & Lipoic acid & $4.70 \mathrm{E}-02$ \\
\hline Thiamine $\mathrm{HCl}$ & $4.00 E+00$ & Phenol red & $1.50 \mathrm{E}+01$ \\
\hline Retinol, all trans(Vitamin A) & $1.00 \mathrm{E}-01$ & CHIR99021 & $1.40 \mathrm{E}+0 \mathrm{C}$ \\
\hline Vitamin $\mathrm{B}_{12}$ & $1.40 E+00$ & 2-mercaptoethanol & $8.17 \mathrm{E}+00$ \\
\hline
\end{tabular}


A

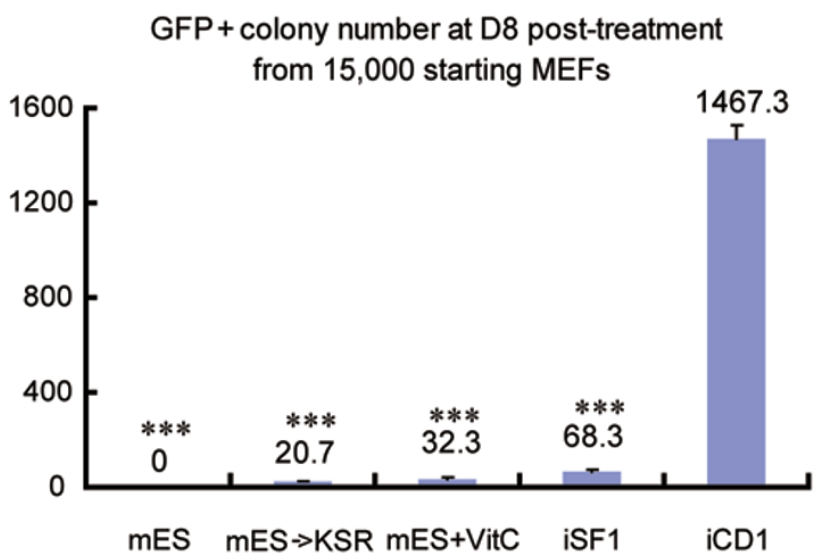

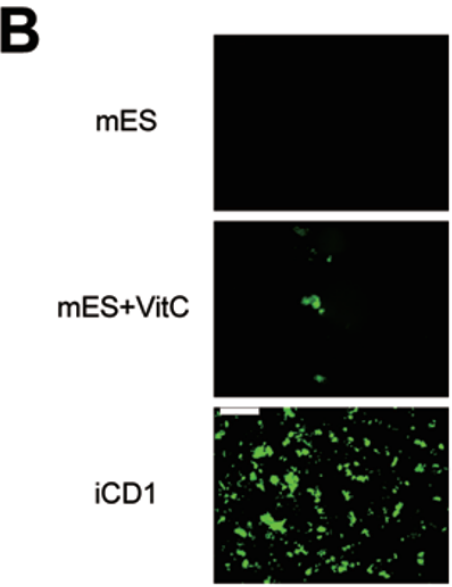

C
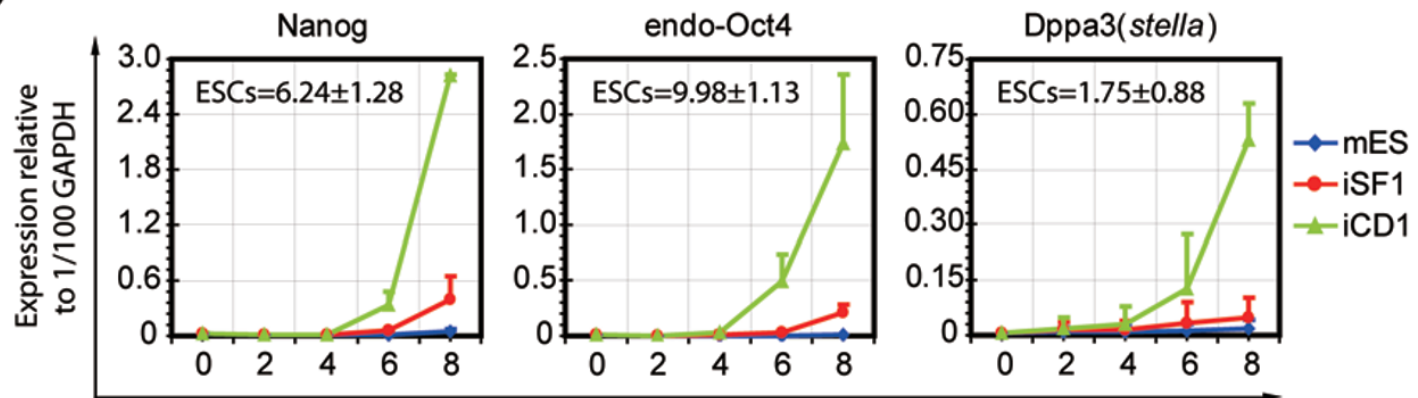

Day post-treatment
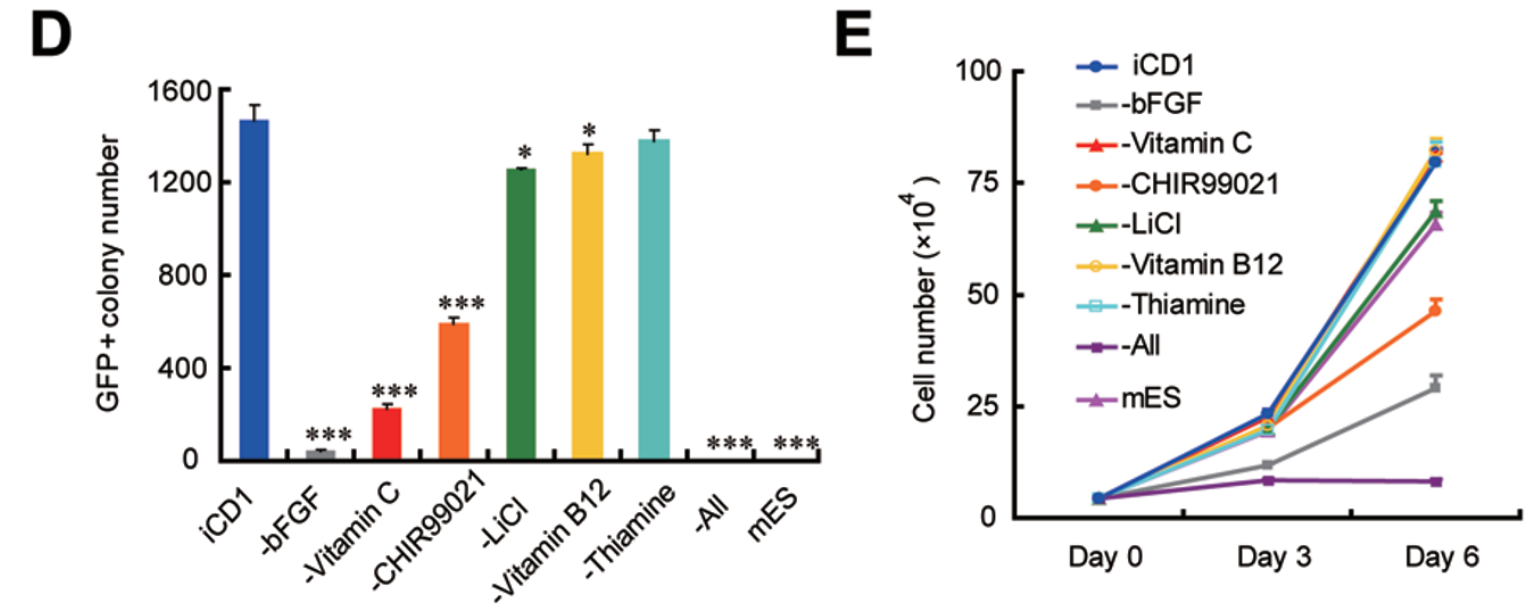

Figure 3 Efficient reprogramming induced by OKS in iCD1. (A) Reprogramming mediated by Oct4/KIf4/Sox2 (OKS) is improved by iCD1. "mES->KSR" indicates the strategy of switching mES medium to KSR medium at day 4 . KSR medium supplemented with bFGF and N2 is called iSF1. Oct4-GFP-positive colonies were scored at day 8 post-treatment from the different culture conditions. $* * * P<0.001$, compared to the result of iCD1 $(n=3)$. (B) Representative fields of OKS-infected MEFs at day 8 post-treatment in the indicated culture conditions. Scale bar: $2 \mathrm{~mm}$. (C) Pluripotent markers are notably activated in iCD1 medium during OSK-mediated reprogramming. Expression levels of Nanog, endogenous Oct4 and Dppa3 were analyzed by qRT-PCR in OSK-infected MEFs cultured in the indicated media on days 0, 2, 4, 6 or 8 post-treatment. Expression values are set relative to GAPDH at $100(n=3)$. (D) MEFs infected with OKS were cultured in mES, iCD1 and iCD1 minus the indicated components. Oct4-GFP colonies were scored at day 8 post-treatment and are shown $(n=3)$. *P< 0.05 and $* * * P<0.001$. (E) Growth curve of MEFs infected with OKS in the indicated media $(n=3)$. 
A

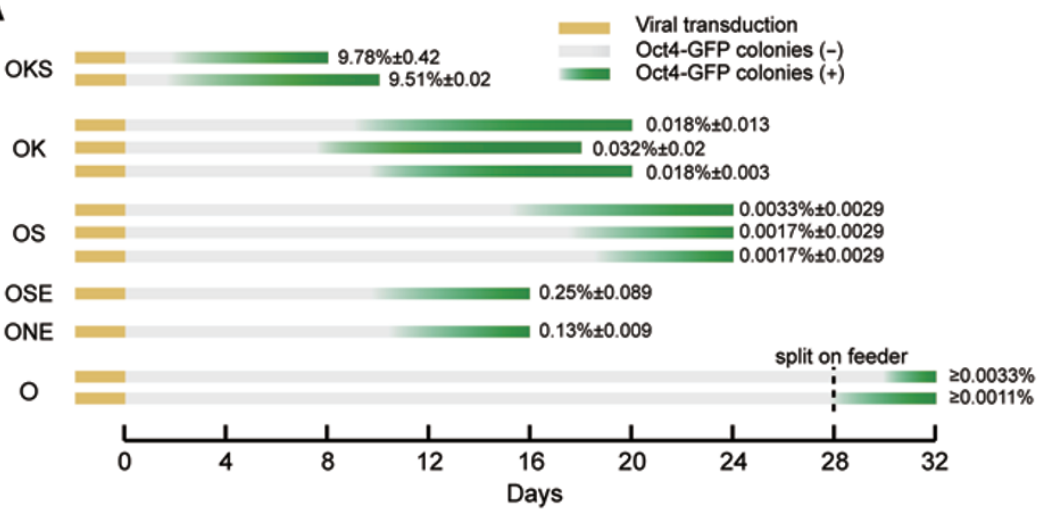

B

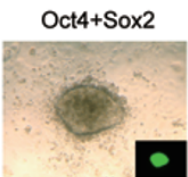

C

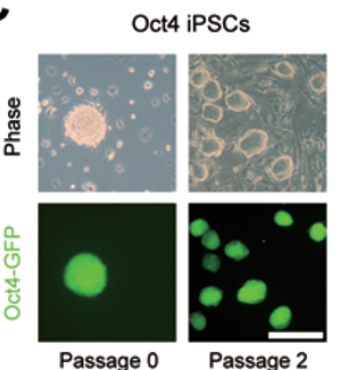

D

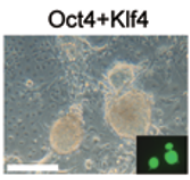

Passage 2
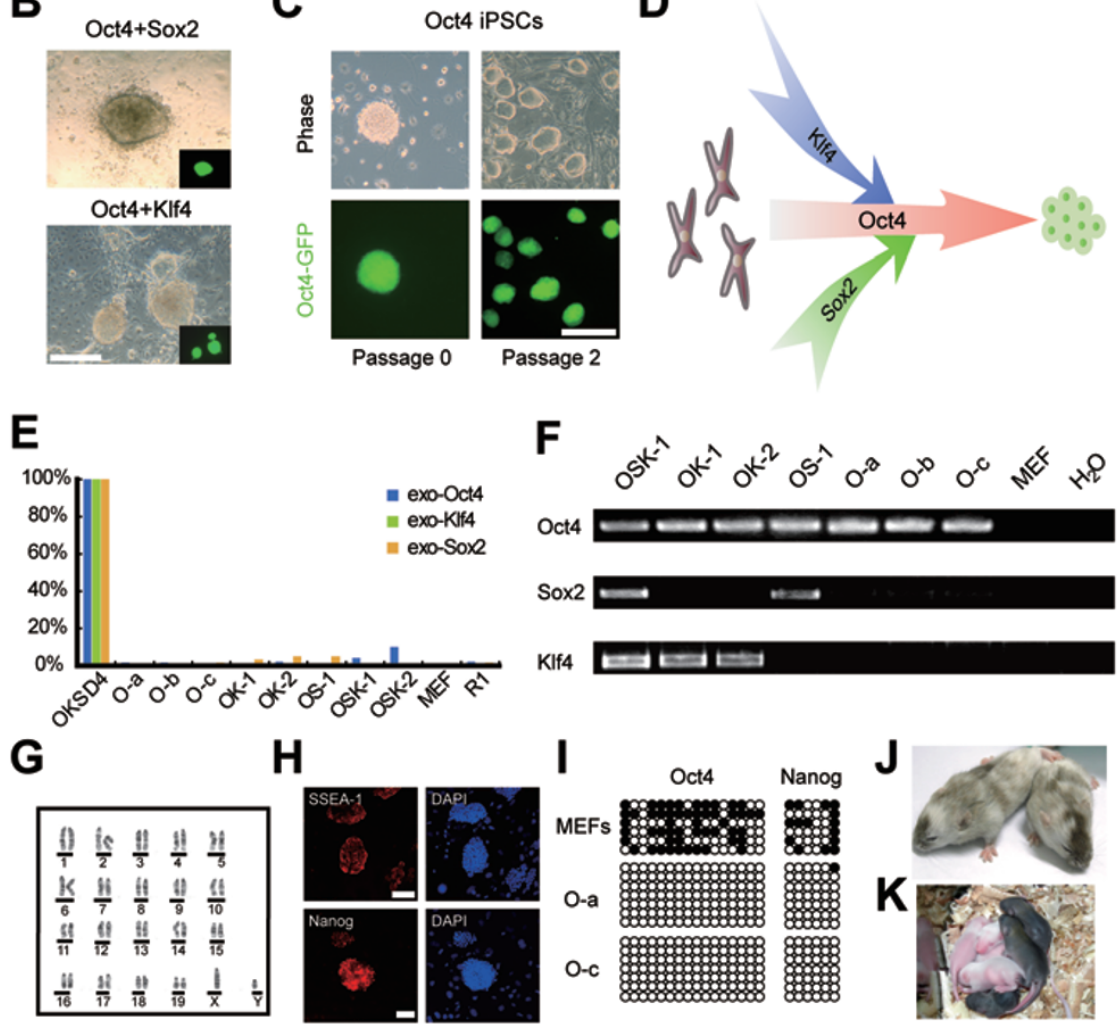

Figure 4 Reprogramming mediated by Oct4 alone in iCD1. (A) Kinetics and efficiency of reprogramming mediated by different factor combinations. Independent experiments were performed with the indicated factors and the Oct4-GFP colonies were scored every other day. Efficiency was determined by the number of Oct4-GFP colonies on the day when the experiments were terminated and are shown as mean \pm SD. Triplicate wells were analyzed for each experiment. (B) Both the combination of Oct4/Sox2 and the combination of Oct4/KIf4 can reprogram MEFs into iPSCs in iCD1. Representative fields show primary colonies that emerged in MEFs infected with Oct4/Sox2 or Oct4/KIf4 cultured in iCD1. Scale bar: $250 \mu \mathrm{m}$. (C) Representative fields show the primary iPSC colony (passage 0) induced from MEFs by Oct4 alone in iCD1 and the iPSC lines derived from this Oct4 iPSC clone (passage 2). Scale bar: $250 \mu \mathrm{m}$. (D) A model depicting the roles of reprogramming factors. Sox2 and Klf4 play auxiliary but important roles in the reprogramming that is triggered by Oct4. (E) Retroviral expression was silenced in iPSC clones. The expression of exogenous Oct4/Sox2/KIf4 was analyzed by qRT-PCR in selected iPSC clones. MEFs infected with OKS for 4 days were used as positive controls. (F) Integration analysis confirms the derivation of OK/OS/ $\mathrm{O}$ iPSC clones induced in iCD1. The presence of the retroviral transgene was examined by PCR. (G) The Oct4 iPSC clones have normal karyotypes. (H) Oct4 iPSCs express SSEA-1 and Nanog (left panels). DAPI staining served as the control (right panels). Scale bar: $100 \mu \mathrm{m}$. (I) Bisulfite sequencing analysis of Oct4 and Nanog promoter regions in Oct4 iPSC clones. Open circles indicate unmethylated $\mathrm{CpG}$ dinucleotides and filled circles indicate methylated CpG dinucleotides. (J) Chimeric mice generated from Oct4 iPSC lines were responsible for the agouti coat color. (K) Germline transmission of chimeric mice generated from Oct4 iPSCs. 
redundant in this optimized condition. We tested whether cMyc would improve the efficiency of OKS-mediated reprogramming and found, surprisingly, that c-Myc only slightly enhanced reprogramming at a low starting cell density (Supplementary information, Figure S4A). We previously reported that the reprogramming process is factor contextdependent [33]. Therefore, it is reasonable that OKS renders better performance with a medium that is optimized specifically for the OKS combination. We also found that VPA can further enhance OKSM-mediated reprogramming in $\mathrm{iCD} 1$, indicating that HDAC activity is a barrier to OKSMmediated reprogramming (Supplementary information, Figure S4B). We also used iCD1 in inducible lentivirus-based reprogramming. The reprogramming efficiency in $\mathrm{iCD} 1$ is very high compared to the traditional medium, while OKSmediated reprogramming is less efficient than the retrovirus system because the lentivirus showed greater cellular toxicity, which would significantly influence reprogramming without c-Myc (Supplementary information, Figure S5A and S5B). We also tested whether iCD1 supports efficient reprogramming in other cell types, including keratinocytes and meningocytes [46] (Supplementary information, Figure S6A and S6B). Compared to other media, iCD1 led to higher OKS-mediated reprogramming efficiency on these two cell types (Supplementary information, Figure S6C, S6D, $\mathrm{S} 6 \mathrm{H}$ and $\mathrm{S} 6 \mathrm{I})$. We also found that using growth medium of keratinocytes on the first 2 days would further enhance reprogramming (Supplementary information, Figure S6D). Resulting iPSC lines expressed pluripotent markers and contributed to chimeric mouse (Supplementary information, Figure S6F, S6G and S6J). Taken together, we concluded that iCD1 represents a new generation of culture media optimized for reprogramming.

\section{Reprogramming of mouse fibroblasts with fewer factors} in $i C D 1$

We then sought to determine the reprogramming efficiencies mediated by fewer factors, namely OS (Oct4/Sox2), OK (Oct4/Klf4) and KS (Klf4/Sox2) in the iCD1. iPSCs were readily generated with OS and OK (Figure 4A and 4B) but not with KS (data not shown). We also examined different combinations, OSE (Oct4/Sox2/Esrrb [47]) and ONE (Oct4/Nanog/Esrrb), and obtained iPSC clones from both (Figure 4A). These results suggest a central role for Oct4 in pluripotency induction. Remarkably, we showed that iPSCs can be generated by a single factor, Oct4, in iCD1 (Figure 4A and 4C), although with slow kinetics (more than 4 weeks) and at low efficiency (Figure 4A). The fact that Oct4 alone can generate iPSCs from MEFs suggests that both Sox 2 and Klf4 are dispensable for reprogramming. We then tried to quantify the relative contributions of Klf4 and Sox 2 under our experimental conditions. We showed that under identical conditions (in iCD1), OKS generates iPSCs at $\sim 10 \%$ in 9 days, OK generates iPCSs at $\sim 0.02 \%$ in 18 days and OS generates iPCSs at $\sim 0.002 \%$ in 24 days (Figure $4 \mathrm{~A})$. Based on this, we can estimate that Sox 2 accelerates reprogramming from $\sim 0.02 \%$ in 18 days to $\sim 10 \%$ in 9 days (or 500-fold plus 9 days). On the other hand, Klf4 leads to a $0.002 \%$ to $10 \%$ increase in efficiency and shortens the time needed by 15 days ( $\sim 5000$-fold plus 15 days). Based on these results, it is apparent that Oct4 alone is sufficient to reestablish pluripotency in MEFs, whereas Klf4 and Sox2 play important but auxiliary roles (Figure 4D).

Klf4 and Sox2, although dispensable for reprogramming in $\mathrm{iCD} 1$, are powerful boosters for reprogramming to yield high efficiency and fast kinetics (Figure 4A). It is interesting that Klf4 appears to be more effective ( $\sim 10$-fold in efficiency and 6 days in kinetics) than Sox 2 . We also examined the endogenous expression of reprogramming factors and found that none of them were significantly induced during the reprogramming process mediated by $\mathrm{OS} / \mathrm{OK} / \mathrm{O}$ in $\mathrm{iCD} 1$ (Supplementary information, Figure S7). Ectopic retroviruses were silenced in these iPSCs, indicating that they maintain pluripotency without transgene expression (Figure 4E). We confirmed that the iPSCs induced by OS/OK/O harbor the respective transgenes by genomic PCR and Southern blot analysis (Figure 4F and Supplementary information, Figure S8A), show normal karyotypes (Figure 4G) and express pluripotent markers (Figure $4 \mathrm{H}$ and Supplementary information, Figure S8B). The Oct4 and Nanog promoter regions were demethylated in these Oct4-iPSC clones (Figure 4I). Expression levels of Gtl2 and Rian in iPSC lines were similar to those in ESCs (Supplementary information, Figure $\mathrm{S} 8 \mathrm{C}$ ). Thus, we speculate that reprogramming in iCD1 would be not only efficient but also complete. The OS/OK/ O-iPSCs contributed to chimeric mice when injected into blastocysts (Figure 4J and Supplementary information, Figure S8D). Furthermore, we obtained germline transmission of Oct4-iPSCs by breeding the chimeras (Figure 4K).

\section{Discussion}

In this study we showed a defined system that enables efficient reprogramming of mouse fibroblasts using a different combination of defined factors. We believe that this reprogramming system can be used as an ideal platform for investigating the individual contribution of medium supplements and reprogramming factors. We found that the function of compounds such as VPA on reprogramming is factor-context dependent, which suggests that the process should be analyzed with different combinations and that only the common mechanism is actually important. Further careful investigation should be performed on context-dependent effect of compounds among different reprogramming models. bFGF, whose recep- 
tor is an RTK, is an unexpected effective component in iCD1. The current data could not exclude the possibility that bFGF affects other cellular processes besides supporting proliferation, as bFGF is also critical for OKSM-mediated reprogramming (Supplementary information, Figure S4C). The MEK1/2 inhibitor greatly inhibits reprogramming, suggesting that activation of the ERK pathway might be important for reprogramming fibroblasts. In addition, this chemically defined system provides safe culture methods for iPSC derivation. Although there is no valid evidence indicating that iPSCs derived in iCD1 would function better than traditionally derived iPCSs, elimination of xeno-contamination and the possibility of improving reprogramming quality highlight the application potential of iCD1.

The ultra-high efficiency and rapid kinetics of OKSmediated reprogramming in iCD1 could become a standard model for the study of iPSC induction mechanisms. Because much more incompletely reprogrammed iPSCs (also named pre-iPSCs) were generated with the transduction of c-Myc $[27,33,41,44]$, the widely used OKSM-mediated reprogramming system may provide inaccurate information of the conversion. However, the low efficiency of OKS-mediated reprogramming restricted its application in mechanistic research. In iCD1, OKS-mediated reprogramming achieved $10 \%$ efficiency within 8 days and induced little pre-iPSCs. Therefore, the critical molecular change that occurred in reprogramming process would be more readily discovered. We also found that many variables, such as cell density, virus family and passage process, would influence final reprogramming efficiency profoundly. It is noticeable that many studies claimed high efficiency by using improper or variable standard to evaluate reprogramming, which would not benefit the mechanistic research. Furthermore, we showed that Oct4, the core factor in ESC transcriptional regulation, is sufficient to reprogram MEFs. The dissection of reprogramming using multiple factors is complex; therefore, one might anticipate that the mechanism of reprogramming can be better understood in the Oct4-mediated reprogramming model. Exploring the conversion induced by a lesser number of factors will help sorting out the exact function of each factor. For instance, based on the advantage of iCD1, we found that BMPs can functionally replace Klf4 by inducing MET and greatly improve O- or OS-mediated reprogramming [28]. Compared to other single Oct4-mediated reprogramming methods reported recently $[29,30], \mathrm{iCD} 1$ or $\mathrm{iCD} 1+\mathrm{BMP}$ has a chemically defined background, which is useful for further characterizing the function of each component while avoiding lot-to-lot variability. The efficiency of O/OS-mediated reprogramming in $\mathrm{ICD} 1+\mathrm{BMP}$, which is the highest in literature, was well defined with multiple independent repeats so that it should be well reproducible. Moreover, signaling transduction plays important roles in cell fate determination, especially during development. As a forced cell fate conversion, reprogramming must profoundly involve signaling transduction. In light of the chemically defined background, iCD1 could serve as a standard system for extracellular signaling study. In conclusion, iCD1 possesses extensive application in future mechanistic dissections of somatic reprogramming.

\section{Materials and Methods}

\section{Cell culture}

Mouse embryonic fibroblasts (MEFs) were derived from E13.5 embryos carrying the Oct4-GFP transgenic allele and maintained in DMEM supplemented with 10\% FBS. iPSCs and ESCs were maintained on MEF feeder layers in both $\mathrm{mES}$ and KSR media. mES medium was prepared as DMEM supplemented with $15 \%$ FBS and LIF (Millipore). KSR medium was prepared as DMEM supplemented with knockout serum replacement (SR) and LIF. iSF1 (DMEM supplemented with SR, N2, LIF and bFGF) [33] and iCD1 (Table 1) were used in iPSC generation. MEF feeder cells were inactivated by mitomycin C. N2, NEAA, bFGF and lipid mixture were purchased from Invitrogen (formulations of NEAA and lipid mixture are available in open information). Corticosterone was purchased from Alexis. Vitamin C (Cat. No. 49752) and other compounds used in iCD1 were purchased from Sigma and CHIR99021 was synthesized at GIBH.

\section{Retrovirus and lentivirus production}

Retroviral vectors (pMXs) containing the murine cDNAs of Oct4, Sox2, Klf4 and c-Myc [4] were purchased from Addgene. These plasmids were transfected into PlatE cells using calcium phosphate transfection. Lentiviruses encoding reprogramming factors under TRE promoter were prepared by transfecting lentivirus vector, psPAX and pMD2G into $293 \mathrm{~T}$ cells and used at MOI $=10$.

\section{Generation of iPS Cells}

MEFs within two passages were split when they reached 80$90 \%$ confluence, plated at 4000 cells $/ \mathrm{cm}^{2}$ for OKS-based iPSC generation or 7500 cells $/ \mathrm{cm}^{2}$ for other combinations $12 \mathrm{~h}$ before infection. The viral supernatants were then collected and filtered $48 \mathrm{~h}$ later to infect MEFs with $4 \mu \mathrm{g} / \mathrm{ml}$ polybrene. The same procedure was repeated once on the following day. The day that viral supernatants were removed and the test media were added was defined as day 0 post-treatment. iPSC colonies were scored or picked 8-10 days (OKS combination) post-treatment in $\mathrm{iCD} 1$ based on the Oct4-GFP expression and the ESC morphology. Photos showing the whole well were taken with SteREO Lumar V12 (Zeiss). Feeder cells were never used in reprogramming procedure. The picked colonies were subsequently expanded and maintained in KSR with feeder layer. A more detailed protocol is available in Supplementary information.

\section{Immunofluorescence staining}

Immunofluorescence staining was performed as previously described [32]. The following primary antibodies were used: goat anti-mNanog, mouse anti-SSEA-1 (R\&D), mouse anti-Oct4 (Abcam) and mouse anti-Rex1 (produced at GIBH). A Leica TCS SP2 spectral confocal microscope was used for immunofluorescence 
studies.

\section{Quantitative RT-PCR}

Total RNA was extracted using TRIzol and $2 \mu \mathrm{g}$ was used to synthesize cDNA with ReverTra Ace ${ }^{\circledR}$ (Toyobo) and oligo-dT (Takara). Quantitative PCR was performed using Premix Ex Taq ${ }^{\mathrm{TM}}$ (Takara) and analyzed with ABI 7300. At least two independent samples were analyzed in this study.

\section{Blastocyst injection}

Chimeras were produced by injecting iPSCs into blastocysts derived from ICR mice, followed by implantation into pseudopregnant ICR mice. F2 mice were breeding with chimeric mice and ICR mice to determine germline transmission of iPSCs.

\section{Bisulfite genomic sequencing}

Genomic DNA (700 ng) from various cell lines was used for bisulfite modification by exposure overnight to a mixture of $50.6 \%$ sodium bisulfite (Sigma S-1516) and $10 \mathrm{mM}$ hydroquinone (Sigma H-9003). The promoter regions of Oct 4 and Nanog were amplified by PCR. The PCR products were cloned into the pMD18-T vector (Takara), propagated and then sequenced.

\section{Derivation of keratinocytes}

Newborn mice were sacrificed and rinsed by iodine tincture, $70 \%$ ethanol and PBS supplemented with penicillin/streptomycin. The skin was peeled off and floated on the surface of $0.25 \%$ trypsin solution at $4{ }^{\circ} \mathrm{C}$ overnight, with the epidermis facing upward. Then the skin was transferred to a sterile surface with the epidermis facing down. The dermis was separated and stirred in KGM2 medium (Lonza) with $1.4 \mathrm{mM}$ calcium chloride to release keratinocytes. The cell suspension was filtered with a $100-\mu \mathrm{m}$ mesh nylon gauze and then plated in gelatin-coated dish in KGM2 medium with 0.2 $\mathrm{mM}$ calcium chloride. Keratinocytes were used for reprogramming in 3 days after derivation. The viruses were collected with lowcalcium serum-free medium (KBM2 supplemented with insulin, EGF) to avoid differentiation of keratinocytes.

\section{Southern blot analysis}

In all, $20 \mu \mathrm{g}$ genomic DNA was digested with EcoRI and BamHI overnight and then separated on a $0.8 \%$ agarose gel. Transfer was performed overnight using the capillary siphon method. Hybridization and detection were performed according to the user manual of DIG High Prime DNA Labeling and Detection Starter Kit II (Roche) with a different hybridization buffer $(10 \%$ PEG6000, 0.1\% SDS, 6× SSC, 50\% formamide and $100 \mathrm{ng} / \mathrm{ml}$ salmon sperm DNA) and CDP-star chemiluminescent substrate (Roche) for detection. Sox 2 and Klf4 full-length cDNA and exon 1 of Oct 4 were used for probe labeling. PCR primers used for Oct4 probe were as follows: $5^{\prime}$-mOct4 (5'-ATGGCTGGACACCTGGCT-3') and 3'-mOct4 exon 1 (5'-CGGGAGTTGGTTCCACCT-3').

\section{Acknowledgments}

We acknowledge Dr Guoliang Xu (Institute of Biochemistry and Cell Biology, Shanghai Institute for Biological Sciences, Chinese Academy of Sciences) for discussion. We are grateful to Jian Zhang and Xia Kuang for assistance with Southern blotting. We are also grateful to Ronghui Li, Jieying Zhu, Jing Li, Keyu Lai, Hengpeng Guo, Shaoxian Sun, Haixiang Zhang, Yi Zheng and Hongwen Pang for their technical assistance. We also thank all members of our laboratory for supporting our work. This work is supported by the National Natural Science Foundation of China (90813033), Key Technologies R\&D Program: the 973 Program of China (2009CB941102 and 2011CB965200), Ministry of Science and Technology International Technology Cooperation Program (2010DFB30430), Chinese Academy of Sciences/SAFEA International Partnership Program for Creative Research Teams, Major Scientific and Technological Special Project: New Drug Innovation and Development (2011X09102-010-01), and "Strategic Priority Research Program" of the Chinese Academy of Sciences (XDA01020401).

\section{References}

1 Evans MJ, Kaufman MH. Establishment in culture of pluripotential cells from mouse embryos. Nature 1981; 292:154-156.

2 Martin GR. Isolation of a pluripotent cell line from early mouse embryos cultured in medium conditioned by teratocarcinoma stem cells. Proc Natl Acad Sci USA 1981; 78:76347638.

3 Thomson JA, Itskovitz-Eldor J, Shapiro SS, et al. Embryonic stem cell lines derived from human blastocysts. Science 1998; 282:1145-1147.

4 Takahashi K, Yamanaka S. Induction of pluripotent stem cells from mouse embryonic and adult fibroblast cultures by defined factors. Cell 2006; 126:663-676.

5 Takahashi K, Tanabe K, Ohnuki M, et al. Induction of pluripotent stem cells from adult human fibroblasts by defined factors. Cell 2007; 131:861-872.

6 Yu J, Vodyanik MA, Smuga-Otto K, et al. Induced pluripotent stem cell lines derived from human somatic cells. Science 2007; 318:1917-1920.

7 Jaenisch R, Young R. Stem cells, the molecular circuitry of pluripotency and nuclear reprogramming. Cell 2008; 132:567582.

8 Scholer HR, Hatzopoulos AK, Balling R, Suzuki N, Gruss P. A family of octamer-specific proteins present during mouse embryogenesis: evidence for germline-specific expression of an Oct factor. EMBO J 1989; 8:2543-2550.

9 Nichols J, Zevnik B, Anastassiadis K, et al. Formation of pluripotent stem cells in the mammalian embryo depends on the POU transcription factor Oct4. Cell 1998; 95:379-391.

10 Niwa H, Miyazaki J, Smith AG. Quantitative expression of Oct-3/4 defines differentiation, dedifferentiation or selfrenewal of ES cells. Nat Genet 2000; 24:372-376.

11 Loh YH, Wu Q, Chew JL, et al. The Oct4 and Nanog transcription network regulates pluripotency in mouse embryonic stem cells. Nat Genet 2006; 38:431-440.

12 Wang J, Rao S, Chu J, et al. A protein interaction network for pluripotency of embryonic stem cells. Nature 2006; 444:364368.

13 Chen $\mathrm{X}, \mathrm{Xu} \mathrm{H}$, Yuan $\mathrm{P}$, et al. Integration of external signaling pathways with the core transcriptional network in embryonic stem cells. Cell 2008; 133:1106-1117.

14 Kim J, Chu J, Shen X, Wang J, Orkin SH. An extended transcriptional network for pluripotency of embryonic stem cells. 
Cell 2008; 132:1049-1061.

15 Avilion AA, Nicolis SK, Pevny LH, Perez L, Vivian N, Lovell-Badge R. Multipotent cell lineages in early mouse development depend on SOX2 function. Genes Dev 2003; 17:126-140.

16 Mitsui K, Tokuzawa Y, Itoh $\mathrm{H}$, et al. The homeoprotein Nanog is required for maintenance of pluripotency in mouse epiblast and ES cells. Cell 2003; 113:631-642.

17 Smith AG, Heath JK, Donaldson DD, et al. Inhibition of pluripotential embryonic stem cell differentiation by purified polypeptides. Nature 1988; 336:688-690.

18 Ying QL, Nichols J, Chambers I, Smith A. BMP induction of Id proteins suppresses differentiation and sustains embryonic stem cell self-renewal in collaboration with STAT3. Cell 2003; 115:281-292.

19 Ying QL, Wray J, Nichols J, et al. The ground state of embryonic stem cell self-renewal. Nature 2008; 453:519-523.

20 Silva J, Nichols J, Theunissen TW, et al. Nanog is the gateway to the pluripotent ground state. Cell 2009; 138:722-737.

21 Ludwig TE, Bergendahl V, Levenstein ME, Yu J, Probasco MD, Thomson JA. Feeder-independent culture of human embryonic stem cells. Nat Methods 2006; 3:637-646.

22 Ludwig TE, Levenstein ME, Jones JM, et al. Derivation of human embryonic stem cells in defined conditions. Nat Biotechnol 2006; 24:185-187.

$23 \mathrm{Xu}$ RH, Sampsell-Barron TL, Gu F, et al. NANOG is a direct target of TGFbeta/activin-mediated SMAD signaling in human ESCs. Cell Stem Cell 2008; 3:196-206.

24 Gurdon JB, Melton DA. Nuclear reprogramming in cells. Science 2008; 322:1811-1815.

25 Pei D. Regulation of pluripotency and reprogramming by transcription factors. $J$ Biol Chem 2009; 284:3365-3369.

26 Maherali N, Sridharan R, Xie W, et al. Directly reprogrammed fibroblasts show global epigenetic remodeling and widespread tissue contribution. Cell Stem Cell 2007; 1:55-70.

27 Nakagawa M, Koyanagi M, Tanabe K, et al. Generation of induced pluripotent stem cells without Myc from mouse and human fibroblasts. Nat Biotechnol 2008; 26:101-106.

28 Chen J, Liu J, Yang J, et al. BMPs functionally replace Klf4 and support efficient reprogramming of mouse fibroblasts by Oct4 alone. Cell Res 2011; 21:205-212.

29 Li Y, Zhang Q, Yin X, et al. Generation of iPSCs from mouse fibroblasts with a single gene, Oct4, and small molecules. Cell Res 2011; 21:196-204.

30 Yuan X, Wan H, Zhao X, Zhu S, Zhou Q, Ding S. Combined chemical treatment enables Oct4-induced reprogramming from mouse embryonic fibroblasts. Stem Cells 2011; doi:10.1002/stem.594

31 Zhu S, Li W, Zhou H, et al. Reprogramming of human primary somatic cells by OCT4 and chemical compounds. Cell
Stem Cell 2010; 7:651-655.

32 Li R, Liang J, Ni S, et al. A mesenchymal-to-epithelial transition initiates and is required for the nuclear reprogramming of mouse fibroblasts. Cell Stem Cell 2010; 7:51-63.

33 Chen J, Liu J, Han Q, et al. Towards an optimized culture medium for the generation of mouse induced pluripotent stem cells. J Biol Chem 2010; 285:31066-31072.

34 Samavarchi-Tehrani P, Golipour A, David L, et al. Functional genomics reveals a BMP-driven mesenchymal-to-epithelial transition in the initiation of somatic cell reprogramming. Cell Stem Cell 2010; 7:64-77.

35 Maherali N, Hochedlinger K. Tgfbeta signal inhibition cooperates in the induction of iPSCs and replaces Sox 2 and cMyc. Curr Biol 2009; 19:1718-1723.

36 Huangfu D, Maehr R, Guo W, et al. Induction of pluripotent stem cells by defined factors is greatly improved by smallmolecule compounds. Nat Biotechnol 2008; 26:795-797.

37 Feng B, Ng JH, Heng JC, Ng HH. Molecules that promote or enhance reprogramming of somatic cells to induced pluripotent stem cells. Cell Stem Cell 2009; 4:301-312.

38 Li W, Zhou H, Abujarour R, et al. Generation of humaninduced pluripotent stem cells in the absence of exogenous Sox2. Stem Cells 2009; 27:2992-3000.

39 Esteban MA, Wang T, Qin B, et al. Vitamin C enhances the generation of mouse and human induced pluripotent stem cells. Cell Stem Cell 2010; 6:71-79.

40 Ichida JK, Blanchard J, Lam K, et al. A small-molecule inhibitor of tgf-Beta signaling replaces sox 2 in reprogramming by inducing nanog. Cell Stem Cell 2009; 5:491-503.

41 Han J, Yuan P, Yang H, et al. Tbx3 improves the germ-line competency of induced pluripotent stem cells. Nature 2010; 463:1096-1100.

42 Chan EM, Ratanasirintrawoot S, Park IH, et al. Live cell imaging distinguishes bona fide human iPS cells from partially reprogrammed cells. Nat Biotechnol 2009; 27:1033-1037.

43 Barnes D, Sato G. Serum-free cell culture: a unifying approach. Cell 1980; 22:649-655.

44 Judson RL, Babiarz JE, Venere M, Blelloch R. Embryonic stem cell-specific microRNAs promote induced pluripotency. Nat Biotechnol 2009; 27:459-461.

45 Hanna J, Saha K, Pando B, et al. Direct cell reprogramming is a stochastic process amenable to acceleration. Nature 2009; 462:595-601.

46 Qin D, Gan Y, Shao K, et al. Mouse meningiocytes express Sox 2 and yield high efficiency of chimeras after nuclear reprogramming with exogenous factors. J Biol Chem 2008; 283:33730-33735.

47 Feng B, Jiang J, Kraus P, et al. Reprogramming of fibroblasts into induced pluripotent stem cells with orphan nuclear receptor Esrrb. Nat Cell Biol 2009; 11:197-203.

(Supplementary information is linked to the online version of the paper on the Cell Research website.) 